\title{
Mutations in the Procaspase-3 Dimer Interface Affect the Activity of the Zymogen†
}

\author{
Cristina Pop $\ddagger$, Brett Feeney $\ddagger$, Ashutosh Tripathy $§$, and A. Clay Clark ${ }^{*}, \ddagger$ \\ Department of Molecular and Structural Biochemistry, North Carolina State University, Raleigh, \\ North Carolina 27695, and Macromolecular Interactions Facility, University of North Carolina at \\ Chapel Hill, Chapel Hill, North Carolina 27599 \\ ‡ North Carolina State University. \\ $\S$ University of North Carolina at Chapel Hill.
}

\begin{abstract}
The interface of the procaspase- 3 dimer plays a critical role in zymogen maturation. We show that replacement of valine 266, the residue at the center of the procaspase- 3 dimer interface, with glutamate resulted in an increase in enzyme activity of $\sim 60$-fold, representing a pseudoactivation of the procaspase. In contrast, substitution of V266 with histidine abolished the activity of the procaspase- 3 as well as that of the mature caspase. While the mutations do not affect the dimeric properties of the procaspase, we show that the V266E mutation may affect the formation of a loop bundle that is important for stabilizing the active site. In contrast, the V266H mutation affects the positioning of loop L3, the loop that forms the bulk of the substrate binding pocket. In some cases, the amino acids affected by the mutations are $>20 \AA$ from the interface. Overall, the results demonstrate that the integrity of the dimer interface is important for maintaining the proper active site conformation.
\end{abstract}

Programmed cell death is dependent on the maturation of the effector caspases from latent zymogens. While the procaspases are present at relatively high concentrations in the cell, it is not clear why they are enzymatically inactive. The mature caspase is a dimer of heterodimeric units containing a large subunit $(17 \mathrm{kDa})$ and a small subunit $(12 \mathrm{kDa})$. In the procaspase monomer, the structural units are organized as a short pro domain (28 amino acids), the large subunit, an intersubunit linker ( 25 amino acids), and the small subunit. Two monomers assemble into the procaspase homodimer that consists of a contiguous 12stranded $\beta$-sheet core with several helices surrounding the $\beta$-sheet (see Figure 1). The procaspase is cleaved at D175, in the intersubunit linker, to remove the covalent connection between the subunits. The pro domain is then removed after cleavage at D9 and then at D28. Recently determined structures of procaspase-7 $(1,2)$ show that the core structural unit of the procaspase is comparable to that of the mature caspase (see Figure 1). The primary differences reside in five active site loops (for a review, see ref 3 ). Following maturation of procaspase-7, only the position of loop L1 (residues 52-67, caspase-3 numbering) remains unaltered. The data show that the procaspase is not enzymatically active because loop L3 (residues 198-213) is unraveled and positioned away from the active site, and the catalytic C163 is rotated away from solvent so that it cannot attack the substrate. The positioning of

\footnotetext{
$\dagger$ This work was supported by a grant from the National Institutes of Health (GM065970) and by the North Carolina Agricultural Research Service.

(C) 2003 American Chemical Society

* To whom correspondence should be addressed: Department of Molecular and Structural Biochemistry, 128 Polk Hall, North Carolina State University, Raleigh, NC 27695-7622. Phone: (919) 515-5805. Fax: (919) 515-2047. clay_clark@ncsu.edu..
} 
C163 is likely due to the covalent connection between loop L2 (residues 163-175) and the intersubunit linker (residues 176-192). Following cleavage at D175, loop L3 moves more than $10 \AA$ toward the protein core to form the substrate binding pocket. Loop L2 moves toward L4, and the intersubunit linker, now called loop L2', flips $180^{\circ}$ to interact with loops L2 and L4 of the second monomer, forming the loop bundle. These movements are summarized in Figure 1.

While the structure of procaspase-7 is thought to represent that of procaspase-3, which is a member of the same caspase subfamily and for which no structural data are available, Nicholson and co-workers (4) showed that procaspase-3 is catalytically competent by using an active site probe that required catalytic turnover to modify the active site. In the accompanying paper (19), we show that the activity of procaspase-3 is $\sim 200$-fold lower than that of the mature caspase- 3 . We further show that while the procaspase binds a substrate with an affinity similar to that of the mature caspase, the lower activity results from a decreased catalytic efficiency. Moreover, results from several biochemical and biophysical studies (19) suggest that in procaspase-3 loop L3 is not unraveled and solvent-exposed, but rather is inserted into the active site. The data suggest a structural model in which the binding pocket is formed but is less solvent accessible in the zymogen, probably because of the presence of the intersubunit linker near the active site. This results in an increase in the $\mathrm{p} K_{\mathrm{a}}$ values of the two catalytic residues, $\mathrm{H} 121$ and C163, relative to the mature caspase- 3 . Upon maturation, that is, cleavage at D175, the active site becomes more solvent accessible because of movements of loops L2 and L4 away from the active site. This is reflected in a decrease in the $\mathrm{p} K_{\mathrm{a}}$ values of $\mathrm{H} 121$ and $\mathrm{C} 163$ and a red shift in tryptophan fluorescence emission from the two tryptophans in the active site.

In addition to significantly stabilizing the protein (5), dimerization is linked to active site formation since it has been shown that the caspase monomer is catalytically inactive (6). Although the mechanism is unknown, the linkage between dimerization and active site formation likely occurs through stabilization of the loop bundle. That is, the interactions between loop L2' and loops L2 and L4 cannot occur in the monomer. The 2-fold symmetry axis of the heterotetramer traverses the V266-V266' interface from each small subunit (Figure 1B), and hydrophobic interactions between the two valine side chains likely contribute to the confor mational stability. We show here that the interface plays a critical role in the zymogen maturation. Replacement of V266 with glutamate resulted in an increase in the activity of procaspase- 3 of $\sim 60$-fold, representing a pseudoactivation of the procaspase without the accompanying polypeptide cleavage. In contrast, substitution of V266 with histidine abolished the enzymatic activity of procaspase-3 as well as that of the mature caspase. We show that the mutations affect the conformations of the active site loops, and in some cases the distances from the interface are >20 ̊.

\section{MATERIALS AND METHODS}

\section{Cloning and Mutagenesis}

Mutants were created using the Quick-Change site-directed mutagenesis kit (Stratagene) and the primers described below. The plasmids used as templates were pHC332, pHC33201, and pHC33209 $(7,19)$, which generated the interface mutations in wild-type caspase-3, procaspase-3(C163S), and procaspase-3(D9A/D28A/D175A), respectively. For the V266H mutants, primers 1 and 2 were used, 5 '-

CAGATTCCATGTATTCATAGCATGCTCACAAAAGAACTC- $3^{\prime}$ and 5'GAGTTCTTTTGTGAGCATGCTATGAATACATGGAATCTG-3', respectively. For V266E mutants, primers 3 and 4 were used, 5'CAGATTCCATGTATTGAGTCCATGCTCACAAAAGAACTC-3' and GAGTTCTTTTGTGAGCATGGACTCAATACATGGAATCTG, respectively. Primers 1 
and 2 introduced a unique $S p h \mathrm{I}$ site (underlined). The mutated bases are shown in bold. The plasmids were sequenced (both strands) to confirm the mutations.

\section{Protein Purification}

Procaspase-3(C163S), caspase-3, procaspase-3( $\left.\mathrm{D}_{3} \mathrm{~A}\right), 1$ and the V266H mutants were purified using previously described protocols $(7,19)$. Caspase-3(V266E) was purified using the protocol described for caspase-3 (19), with the following modifications. The cells were grown at $25^{\circ} \mathrm{C}$, induced with $0.5 \mathrm{mM}$ IPTG at an $A_{600}$ of $\sim 1.2$, and harvested after induction for $4 \mathrm{~h}$. For procaspase-3(V266E), Escherichia coli BL21(DE3) TUNER cells were used. The cells were grown at $20^{\circ} \mathrm{C}$, induced with $0.4 \mathrm{mM}$ IPTG at an $A_{600}$ of $\sim 1.2$, and harvested after induction of $18 \mathrm{~h}$. The remaining purification was carried out as described in ref 19 .

\section{Analytical Ultracentrifugation}

Sedimentation equilibrium experiments were performed as described previously (7). The proteins [in $50 \mathrm{mM}$ phosphate $(\mathrm{pH} 7.2)$ and $1 \mathrm{mM}$ DTT] were at the following concentrations: (A) 11.3, 18.8, and $26.4 \mu \mathrm{M}$ procaspase-3(C163S/V266E), (B) 7.5, 18.8, and $30.1 \mu \mathrm{M}$ procaspase-3(C163S/V266H), (C) 5.6 and $15.0 \mu \mathrm{M}$ caspase-3(V266E). The calculated partial specific volumes of the proteins $[0.713 \mathrm{~mL} / \mathrm{mg}$ for procaspase-3(C163S/ $\mathrm{V} 266 \mathrm{E})$ and procaspase-3(C163S/V266H) and $0.714 \mathrm{~mL} / \mathrm{mg}$ for caspase-3(V266E)] were used in the analysis. In separate experiments, caspase-3(V266E) was examined in the presence of the caspase inhibitor Z-VAD-FMK at a caspase:inhibitor molar ratio of 1:5. The experimental data were fit globally using the ORIGIN version (8) of the NONLIN (9) algorithm supplied by Beckman.

\section{DMS Cross-Linking}

Cross-linking with dimethyl suberimidate (DMS) was carried out as described previously (10). Briefly, the proteins were diluted in $0.2 \mathrm{M}$ triethanolamine $(\mathrm{pH} 8.5)$ so that the final protein concentrations were between 10 and $25 \mu \mathrm{M}$. DMS was dissolved in the same buffer and was added at a final molar ratio of $\sim 1: 10$ (protein:DMS). The reaction mixtures were incubated at room temperature for $1 \mathrm{~h}$, and the samples were analyzed by SDS-PAGE (4 to $25 \%$ gradient).

\section{Immunoblotting}

Proteins were resolved on 4 to $25 \%$ SDS-PAGE and then were transferred to a PVDF membrane overnight (11). The Western blot was performed as described in ref 19 except that the secondary antibody (\#SAB300, Stressgen) was HRP-coupled and was used at final dilution of 1:7000. The detection was performed using the ECL detection kit (Amersham) following the manufacturer's instructions.

\section{Enzyme Activity Assay}

Enzyme activity was determined in a buffer containing $20 \mathrm{mM}$ Pipes, $100 \mathrm{mM} \mathrm{NaCl}, 0.1 \%$ CHAPS, $10 \%$ sucrose, and $10 \mathrm{mM}$ DTT (enzyme assay buffer) at $25^{\circ} \mathrm{C}$, as described previously (12). The total reaction volume was $200 \mu \mathrm{L}$, and the final concentration of the enzyme was either $1 \mathrm{nM}$ (caspase-3), $5 \mathrm{nM}$ (V266E mutants), or $10 \mathrm{nM}$ [procaspase-3( $\left.\left.\mathrm{D}_{3} \mathrm{~A}\right)\right]$.

\footnotetext{
${ }^{1}$ Abbreviations: IPTG, isopropyl $\beta$-D-thiogalactopyranoside; TLCK, $N$ - $\alpha$ - $p$-tosyl-L-lysine chloromethyl ketone; TPCK, $N$-tosyl-Lphenylalanine chloromethyl ketone; DMS, dimethyl suberimidate; Z-VAD-FMK, $N$-benzyloxycarbonyl-Val-Ala-Aspfluoromethylketone; Z-DEVD-AFC, $N$-benzyloxycarbonyl-Asp-Glu-Val-Asp-7-amino-4-trifluoromethyl coumarin DFP, diisofluorophosphate; procaspase-3(D3A), procaspase-3(D9A/D28A/D175A).
} 
Digestion of procaspase-3(V266H) $(5.6 \mu \mathrm{M})$ with granzyme B (Calbiochem) was carried out in enzyme assay buffer at $37^{\circ} \mathrm{C}$ for $2 \mathrm{~h}$ at a final caspase:granzyme B molar ratio of 90:1. Following digestion with granzyme $\mathrm{B}$, the initial velocity was measured using final enzyme concentrations of $1 \mathrm{nM}$ (caspase-3) or $10 \mathrm{nM}$ [caspase-3(V266H)] in the presence of $25 \mu \mathrm{M}$ Ac-DEVD-AFC substrate.

Enzyme activity was determined over the $\mathrm{pH}$ range of 5-10 as described previously $(13,19)$. The following buffers were used: $50 \mathrm{mM}$ citrate (pH 3.0-6.2), $20 \mathrm{mM}$ Pipes (pH 6.1-7.5), 50 $\mathrm{mM}$ Tris- $\mathrm{HCl}$ (pH 7.2-9.0), $50 \mathrm{mM}$ bicine (pH 7.6-9.0), $100 \mathrm{mM}$ glycine ( $\mathrm{pH}$ 8.6-10.6), and $50 \mathrm{mM}$ sodium bicarbonate $(\mathrm{pH} 9.2-10.6)$. All experiments were carried out at $25^{\circ} \mathrm{C}$, and all buffers contained $\mathrm{NaCl}, \mathrm{CHAPS}$, sucrose, and DTT as described above for the enzyme assay buffer. Final protein concentrations were $2.5 \mathrm{nM}$ (caspase-3), $1.25 \mathrm{nM}$ (V266E mutants), or $10 \mathrm{nM}$ [procaspase- $\left.3\left(\mathrm{D}_{3} \mathrm{~A}\right)\right]$. The data were analyzed as described in ref 19 using eq 1 :

$$
\log Y=C\left(1+\left[\mathrm{H}^{+}\right] / K_{\mathrm{a} 1}+K_{\mathrm{a} 2} /\left[\mathrm{H}^{+}\right]\right)
$$

where $Y$ is the initial velocity measured at each $\mathrm{pH}, C$ is the $\mathrm{pH}$-independent value of $Y$, and $K_{\mathrm{a} 1}$ and $K_{\mathrm{a} 2}$ are the dissociation constants for the enzyme groups (14).

\section{Trypsin and V8 Protease Digestion}

Limited proteolysis studies were performed as described in ref 19 , and the reactions were stopped with either TLCK $(125 \mu \mathrm{g} / \mathrm{mL})$ or DFP $(180 \mu \mathrm{g} / \mathrm{mL})$ for trypsin or V8 proteases, respectively. The products were analyzed by MALDI-TOF mass spectrometry and by SDSPAGE using 4 to $25 \%$ or 10 to $25 \%$ polyacrylamide gels $(7,19)$. The five $\mathrm{N}$-terminal amino acids of each peptide were determined by Edman degradation (Protein Structure Core Facility, University of Nebraska,, Lincoln, NE).

\section{Acrylamide and Potassium lodide Quenching}

Quenching studies were carried out as described in ref 19. Briefly, acrylamide (5 M stock) or potassium iodide (2 M stock) was added to the protein $(2 \mu \mathrm{M})$ in a buffer of $50 \mathrm{mM}$ potassium phosphate (pH 7.2) and $1 \mathrm{mM}$ DTT at $25^{\circ} \mathrm{C}$ to yield the concentrations shown in the figures. The proteins were excited at $295 \mathrm{~nm}$, and the emission was monitored between 300 and $400 \mathrm{~nm}$ (PTI C-61 spectrofluorometer, Photon Technology International). For pHdependent quenching studies, the following buffers were used: $50 \mathrm{mM}$ sodium citrate $(\mathrm{pH}$ 3-6.5), $50 \mathrm{mM}$ potassium phosphate ( $\mathrm{pH} 6.0-8.0$ ), or $50 \mathrm{mM}$ Tris- $\mathrm{HCl}$ (pH 7.2-9.5). All buffers contained $1 \mathrm{mM}$ DTT.

For acrylamide quenching, the data were fit to the Stern-Volmer equation, accounting for static and dynamic quenching (15). For quenching by iodide, the percent quenching at 345 $\mathrm{nm}\left[\left(\Delta F / F_{0}\right) \times 100\right]$ was plotted versus KI concentration, and the data were fit as described in ref 19 to determine the Stern-Volmer quenching constant $\left(K_{\mathrm{SV}}\right)$. For $\mathrm{pH}$ studies, values of $K_{\mathrm{SV}}$ were plotted versus $\mathrm{pH}$, and the data were fit as described in ref 19 using eq 2

$$
K_{\mathrm{SV}}=A+\Delta K_{\mathrm{SV}_{1}} \times 10^{n_{1}\left(\mathrm{pH}-\mathrm{p} K_{\mathrm{a} 1}\right)} /\left[1+10^{n_{1}\left(\mathrm{pH}-\mathrm{p} K_{\mathrm{a} 1}\right)}\right]+\Delta K_{\mathrm{SV}_{2}} \times 10^{n_{2}\left(\mathrm{pH}-\mathrm{p} K_{\mathrm{a} 2}\right)} /\left[1+10^{n_{2}\left(p H-\mathrm{p} K_{\mathrm{a} 2}\right)}\right]
$$

where $K_{\mathrm{SV}}$ is the Stern-Volmer constant, $A$ is the value of $K_{\mathrm{SV}}$ at the lowest $\mathrm{pH}, \Delta K_{\mathrm{SV}} 1$ and $\Delta K_{\mathrm{SV}}$ are the changes in Stern-Volmer constants for each transition, $n_{1}$ and $n_{2}$ are cooperativity parameters for each transition, and $\mathrm{p} K_{\mathrm{a} 1}$ and $\mathrm{p} K_{\mathrm{a} 2}$ are the apparent $\mathrm{p} K_{\mathrm{a}}$ values of the transitions. 


\section{Fluorescence Emission versus pH}

Proteins $(1 \mu \mathrm{M})$ were incubated in $20 \mathrm{mM}$ sodium citrate (pH 2.5-6.2) or $20 \mathrm{mM}$ potassium phosphate (pH 6.0-9.0) containing $1 \mathrm{mM}$ DTT at $25^{\circ} \mathrm{C}$ for at least $1 \mathrm{~h}$. Samples were excited at $280 \mathrm{~nm}$, and the fluorescence emission was measured between 305 and $400 \mathrm{~nm}$ (PTI C-61 spectrofluorometer). All measurements were corrected for a background signal. For each sample, the average emission wavelength, $\langle\lambda\rangle$, was calculated as described previously (16). Plots of $\langle\lambda\rangle$ versus $\mathrm{pH}$ were fit to eq 3 as described in ref 19

$$
\langle\lambda\rangle=A+\sum_{i=1}^{3}\left[\Delta\langle\lambda\rangle_{i} \times 10^{n_{i}\left(\mathrm{pH}-\mathrm{p} K_{\mathrm{ai}}\right)} /\left(1+10^{n_{i}\left(\mathrm{pH}-\mathrm{p} K_{\mathrm{ai}}\right)}\right)\right]
$$

where $A$ is the value of $\langle\lambda\rangle$ at $\mathrm{pH}>8.0, \Delta\langle\lambda\rangle_{i}$ is the change in average emission wavelength for the corresponding transition, $n_{i}$ is the cooperativity parameter for each transition, and $\mathrm{p} K_{\mathrm{a} i}$ refers to the apparent $\mathrm{p} K_{\mathrm{a}}$ values of the transitions. For all proteins, the results shown in Figure 5 were identical when the proteins were titrated from $\mathrm{pH} 9$ to 2.5 or from $\mathrm{pH} 2.5$ to 9 , demonstrating the reversibility of the transitions.

\section{Circular Dichroism Spectroscopy}

Circular dichroism was measured using a Jasco J600A spectropolarimeter as described previously (7).

\section{RESULTS}

\section{Interface Mutants}

The V266E or V266H mutation was introduced into procaspase-3 and mature caspase-3. In addition, the mutations in procaspase-3 were placed in the background of $\mathrm{C} 163 \mathrm{~S}$ or the D9A/D28A/D175A triple mutant. As described previously, the C163S mutation provides an inactive procaspase- 3 , while the three processing sites remain intact (7). In contrast, the D9A/D28A/D175A background (19), called procaspase-3( $\left.\mathrm{D}_{3} \mathrm{~A}\right)$, removes the three processing sites in procaspase-3, while the active site remains intact. This background provides an enzymatically active, uncleavable procaspase-3 (19).

\section{Interface Mutants Remain Dimeric}

Using analytical ultracentrifugation (AUC) techniques, we have shown that procaspase-3 is a dimer in solution, and we estimate the equilibrium dissociation constant to be less than 50 $\mathrm{nM}$ (7). We used sedimentation equilibrium studies to examine the effect of the interface mutations on the oligomeric properties of the caspases (see Table 1). For each protein, the data were best fit to a single, thermodynamically ideal species that corresponds to a dimer. As shown in Table 1, caspase-3(V266E) also was analyzed in the presence of the covalently bound inhibitor, Z-VAD-FMK. In this experiment, the protein was incubated in the presence of an inhibitor:caspase molar ratio of 5:1. Activity measurements before and after incubation showed a complete loss of enzyme activity, demonstrating that the inhibitor was bound (not shown). While we have not examined procaspase- $3\left(D_{3} A\right)$ (with or without the interface mutations) by AUC, we have performed cross-linking experiments with DMS to examine the oligomeric properties of procaspase- $3\left(\mathrm{D}_{3} \mathrm{~A}\right)$, procaspase-3( $\left.\mathrm{D}_{3} \mathrm{~A} / \mathrm{V} 266 \mathrm{H}\right)$, and procaspase-3( $\left.\mathrm{D}_{3} \mathrm{~A} / \mathrm{V} 266 \mathrm{E}\right)$. As in the background of C163S, the data (not shown) demonstrate that the proteins are dimers. From these studies, we conclude that neither interface mutation affects the oligomeric properties of the procaspases (in the background of $\mathrm{C} 163 \mathrm{~S}$ or $\mathrm{D}_{3} \mathrm{~A}$ ) or of the mature caspases. 
In our previous experiments with procaspase-3(C163S) (7), it was not possible to determine the equilibrium dissociation constant $\left(K_{\mathrm{d}}\right)$ accurately because the monomer is not populated under the conditions of the AUC experiments, even at the lowest protein concentration $(\sim 450 \mathrm{nM})$. This is also true for the mutants. Thus, while it is formally possible that the interface mutations affect the $K_{\mathrm{d}}$, the proteins remain dimeric at protein concentrations of $>50 \mathrm{nM}$.

\section{Enzyme Assays}

We examined the initial velocity over a range of substrate concentrations for the V266E interface mutants, and the results are shown in Figure 2A, in which the data are compared to those of caspase-3. The steady-state parameters $\left(K_{\mathrm{m}}\right.$ and $\left.k_{\mathrm{cat}}\right)$ determined from the data in Figure $2 \mathrm{~A}$, are presented in Table 2 . Results for procaspase-3( $\left.\mathrm{D}_{3} \mathrm{~A}\right)$ are described in ref 19 , and we summarize the results in Table 2 to compare them with the results of the interface mutants. The data show that the values of $K_{\mathrm{m}}$ are similar for all proteins, between $\sim 2$ and $\sim 6$ $\mu \mathrm{M}$. However, as shown in Table 2 , the catalytic efficiency $\left(k_{\text {cat }}\right)$ is $\sim 60$-fold higher for procaspase-3( $\left.\mathrm{D}_{3} \mathrm{~A} / \mathrm{V} 266 \mathrm{E}\right)$ than for procaspase- $3\left(\mathrm{D}_{3} \mathrm{~A}\right)$. This results in a pseudoactivation of the procaspase because the activity is only $\sim 2$-fold lower than that of the mature caspase- 3 , without the corresponding cleavage of the polypeptide chain. Interestingly, there was no further increase in $k_{\text {cat }}$ for caspase-3(V266E). Overall, the specificity constant $\left(k_{\mathrm{cat}} / K_{\mathrm{m}}\right)$ is $\sim 5$-fold lower in the V266E mutants than in the mature caspase-3.

We examined the $\mathrm{pH}$ dependence to the enzyme activity, and the results are shown in Figure $2 \mathrm{~B}$. Like the mature caspase- $3(13,19)$, the activity of procaspase- $3\left(\mathrm{D}_{3} \mathrm{~A}\right)$ displays a bellshaped profile when measured versus $\mathrm{pH}$. As described in ref 13 , this is consistent with the protonation and/or deprotonation of the two catalytic groups, H121 and C163, leading to one active form of the enzyme. It is thought that the catalytic groups reside in the doubly ionized form, $\mathrm{S}^{-} \mathrm{Im}^{+}$. As shown in Figure 2B, a bell-shaped profile was obtained also for the $\mathrm{pH}$ dependent activity of procaspase-3( $\left.\mathrm{D}_{3} \mathrm{~A} / \mathrm{V} 266 \mathrm{E}\right)$ and caspase-3(V266E). The data were fit to eq 1, as described in Materials and Methods, to determine the $\mathrm{p} K_{\mathrm{a}}$ values of the two transitions, and the results are presented in Table 2. For the two V266E mutants, the data show that $\mathrm{p} K_{\mathrm{a} 1}$, presumably the protonation of $\mathrm{C} 163$ (17), decreased by at least $0.5 \mathrm{pH}$ unit when compared to that of procaspase- $3\left(\mathrm{D}_{3} \mathrm{~A}\right)$ so that it was similar to that of wild-type caspase-3. In contrast, $\mathrm{p} K_{\mathrm{a} 2}$, presumably the deprotonation of H121 (17), remained similar to that of the procaspase- 3 . These properties result in broad optimal $\mathrm{pH}$ ranges for both precursor and mature caspase-3(V266E) (Figure 2B and Table 2). Overall, the data suggest that the V266E mutation increases the activity of the procaspase because it affects the environment of C163. In addition, the data suggest that the environment of H121 is similar to that of the procaspase, even in the processed protein.

\section{Characterization of V266E and V266H Interface Mutants}

While it is possible that the activity observed for procaspase-3( $\left.\mathrm{D}_{3} \mathrm{~A} / \mathrm{V} 266 \mathrm{E}\right)$ is due to contaminating mature caspase generated at alternate cleavage sites in the intersubunit linker, this seems unlikely for two reasons. First, we have shown that the activity of procaspase-3( $\left.\mathrm{D}_{3} \mathrm{~A}\right)$ does not result from alternatively processed protein (19). Second, the level of activity of procaspase-3( $\left.\mathrm{D}_{3} \mathrm{~A} / \mathrm{V} 266 \mathrm{E}\right)$ suggests that most of the protein would be processed. To examine this, we performed a Western analysis using an antibody that recognizes the large subunit of caspase-3, and the results are shown in Figure 2C. In this experiment, we examined procaspase- $3\left(\mathrm{D}_{3} \mathrm{~A}\right)$, procaspase- $3\left(\mathrm{D}_{3} \mathrm{~A} / \mathrm{V} 266 \mathrm{E}\right)$, and procaspase-3( $\left.\mathrm{D}_{3} \mathrm{~A} / \mathrm{V} 266 \mathrm{H}\right)$ (lanes 1-3, respectively; 500-600 $\mathrm{ng}$ of protein). The results are compared to three concentrations of mature caspase-3, from 670 to $13 \mathrm{ng}$ (lanes 4-6). Overall, the data show no evidence of the processed large subunit in these samples and 
further demonstrate that the activity of procaspase-3( $\left.\mathrm{D}_{3} \mathrm{~A} / \mathrm{V} 266 \mathrm{E}\right)$ is not due to the contaminating processed protein.

In contrast to the V266E interface mutants, both procaspase-3(V266H) and procaspase-3( $\left.\mathrm{D}_{3} \mathrm{~A} / \mathrm{V} 266 \mathrm{H}\right)$ were enzymatically inactive. During purification, procaspase-3(V266H) remained mostly unprocessed, and the activity of this mutant was $\sim 0.02 \%$ of that of wild-type caspase-3 (data not shown). We attribute this residual activity to the small amount of processed caspase-3(V266H) formed during purification. To obtain the mature caspase-3(V266H), we treated the sample with granzyme B, which cleaves the procaspase at D175 in the intersubunit linker. The fully processed caspase-3(V266H) displayed an enzymatic activity of $\sim 1 \%$ of that of caspase- 3 (data not shown). Overall, the results demonstrate that the effect of the $\mathrm{V} 266 \mathrm{H}$ mutation on enzyme activity is not limited to the procaspase form of the protein.

\section{Limited Trypsin Proteolysis}

We examined the positioning of the active site loops using limited proteolysis with trypsin and V8 proteases. In these studies, we used the enzymatically inactive forms of the procaspases, C163S, although the results were the same for the interface mutants in the context of $\mathrm{C} 163 \mathrm{~S}$ or $\mathrm{D}_{3} \mathrm{~A}$. In the accompanying paper (19), we showed that both procaspase-3(C163S) and mature caspase-3 are cleaved by trypsin in two of the active site loops. Discrete cleavages occur at K57 and R64, in loop L1, and R207, in loop L3 (see Figure 1B). Furthermore, we showed that cleavage at R207 occurred more slowly than the cleavage at K57/R64 and that R207 was protected in the presence of an inhibitor (19).

The fragments generated by trypsin digestion of the interface mutants, procaspase-3(C163S/ $\mathrm{V} 266 \mathrm{H})$ and procaspase-3(C163S/V266E), are shown in Figure 3. As described in ref 19, the cleavage sites were identified by MALDI-TOF mass spectrometry and by peptide sequencing, allowing an unambiguous assignment of the cleavage sites. The results demonstrate that the V266H mutant (Figure 3A) was cleaved rapidly, and the full-length protein was cleaved within the initial 2 min of the experiment. Further analysis showed that the rapid cleavage occurred at R207, producing two fragments of 23 and $9 \mathrm{kDa}$. The $23 \mathrm{kDa}$ fragment is observed as a minor cleavage product in procaspase-3(C163S) (see Figure 4A in ref 19). In a somewhat slower reaction, the $23 \mathrm{kDa}$ fragment was cleaved at R64, generating a $16 \mathrm{kDa}$ fragment. These results are in contrast with those of procaspase-3(C163S) (19), where cleavage occurred first at R64, generating a fragment of $25 \mathrm{kDa}$, followed by cleavage at R207. In the $\mathrm{V} 266 \mathrm{H}$ mutant, the $25 \mathrm{kDa}$ fragment is observed to be a minor component (Figure 3A). Also, in contrast to procaspase-3, there was no protection of R207 in the presence of an inhibitor (not shown), but on the basis of the activity studies, it is likely that the mutant does not bind the inhibitor. Overall, the results suggest that R207 in loop L3 is hyperexposed in procaspase-3(C163S/V266H) relative to its position in procaspase-3(C163S).

In procaspase-3(C163S/V266E) (Figure 3B), trypsin cleaved first at K19 in the pro domain, generating a $30.5 \mathrm{kDa}$ fragment, and then a second cleavage occurred at R64, which generated a $25 \mathrm{kDa}$ fragment. The kinetics of cleavage at R64 were significantly slower than those observed for procaspase-3(C163S), where $t_{1 / 2}$ was shown to be $<2$ min under the conditions of these experiments (19). This suggests that R64 is less accessible to the protease in procaspase-3(C163S/V266E) than in procaspase-3(C163S). In addition, there was no cleavage at R207 in procaspase-3(C163S/V266E), suggesting that this residue is inaccessible to the trypsin. In the presence of the caspase-3 inhibitor, DEVD-CHO, R64 was protected (not shown). In addition to the procaspase, the results for the cleavage of mature caspase-3(V266E) with trypsin were the same as for the procaspase-3(C163S/V266E) (not shown). That is, there was a slow cleavage at R64 in the large subunit and little or no 
cleavage at R207 in the small subunit. Therefore, in this assay, the two V266E mutants exhibit similar properties. Overall, the data suggest that the V266E mutation affects the positions of active site loops L1 and L3 in the procaspase, rendering them less accessible to trypsin.

\section{Limited V8 Proteolysis}

We show in ref 19 that $\mathrm{V} 8$ protease cleaves procaspase-3(C163S) at residues D9, E25, E98, E106, E173, D190, E248, and D253. In Figure 1B, these sites are mapped onto the structure of caspase-3. Residues E248 and D253 reside in loop L4, whereas E173 and D190 are in loops L2 and L2', respectively. Residues E98 and E106 are on the surface of the protein away from the active site, whereas D9 and E25 are in the pro domain (and not shown in the figure). Experimentally, the data demonstrate four closely spaced bands between 32 and 27 $\mathrm{kDa}$, termed bands 1-4. Band 1 represents the full-length protein. Cleavages at E248/D253, in loop L4, generate two fragments of 29 (band 2) and $4 \mathrm{kDa}$. Band 2 is cleaved further at D9, to give band 3, and E25, to give band 4 . Band 4 is then cleaved simultaneously at positions $98 / 106$ and $173 / 190$, giving rise to a mixture of $\sim 16$ and $\sim 8.5 \mathrm{kDa}$ bands.

The time course analysis of V8 digestion of procaspase-3(C163S/V266H) at $\mathrm{pH} 7$ is shown in Figure 3C, and demonstrates the same cleavage pattern observed for the procaspase-3(C163S), described above. As with the trypsin proteolysis studies described above, we used MALDI-TOF mass spectrometry and peptide sequencing to determine the cleavage sites. The results show that the cleavage sites are the same in procaspase-3(C163S/ V266H) and procaspase-3(C163S), with similar kinetics. Overall, the data suggest that there are no differences in loop L4 (E248/D253) or the intersubunit linker (E173/D190) for procaspase-3(C163S/V266H) compared to that of procaspase-3(C163S).

We also have examined the conformation of procaspase-3(C163S/V266E) by limited V8 proteolysis, and the results are shown in Figure 3D. The data demonstrate a cleavage pattern different from that observed for procaspase-3(C163S) or the V266H interface mutant (Figure 3C). For procaspase-3(C163S/V266E), V8 protease cleaved the $\mathrm{N}$-terminus (E25) and then the C-terminus (E272), to generate two fragments of $\sim 30$ and $\sim 26 \mathrm{kDa}$. Importantly, there are no cleavages observed for the E98/E106, E173/E190, or E248/D253 sites, even after prolonged incubation. These results suggest a conformational change that affects loop L4 (E248/D253), the intersubunit linker (E173/E190), and the region of E98/ E106.

\section{Fluorescence Quenching}

As shown in Figure 1, each monomer of the procaspase contains two tryptophanyl residues, W206 and W214, and both reside in the active site. To further examine loop L3, which contains W206, in the interface mutants, we assessed the quenching of fluorescence emission upon the addition of potassium iodide. In these experiments, the percent quenching was plotted versus the concentration of quencher, and the data were fit to a modified form of the Stern-Volmer equation to obtain the quenching constant, $K_{\mathrm{SV}}(19)$. The values of $K_{\mathrm{SV}}$ were determined over the $\mathrm{pH}$ range of 3-9.5, and the results are summarized in Figure 4A [procaspase-3(C163S/V266E) and caspase-3(V266E)] and Figure 4B [procaspase-3(C163S/ $\mathrm{V} 266 \mathrm{H})$ and caspase-3(V266H)]. The data for procaspase-3(C163S) and caspase-3 are given in ref 19 .

The data for procaspase-3(C163S/V266E) are described by two transitions. The first transition occurs between $\mathrm{pH} 3$ and 5 and results in a decrease in $K_{\mathrm{SV}}$, whereas the second transition occurs between $\mathrm{pH} 6$ and 7 and results in an increase in $K_{\mathrm{SV}}$. Similar results were obtained for the wild-type mature caspase-3, where we showed that $K_{\mathrm{SV}}$ increased from $\sim 6$ 
to $\sim 8$ between $\mathrm{pH} 6$ and $9(19)$. The $\mathrm{p} K_{\mathrm{a}}$ values for the transitions, determined by fitting the data to eq 2 , were $4.0 \pm 0.4$ and $6.2 \pm 0.2$, respectively. In contrast, the data for the mature caspase-3(V266E) are described by three transitions. As for the procaspase, the first transition occurs between $\mathrm{pH} 3$ and $5\left(\mathrm{p} K_{\mathrm{a}}=4.2 \pm 0.5\right)$ and results in a decrease in iodide accessibility. The second transition occurs between $\mathrm{pH} 5$ and $6\left(\mathrm{p} K_{\mathrm{a}}=5.5 \pm 0.1\right)$, where $K_{\mathrm{SV}}$ increases to $\sim 10$. The third transition occurs at $\mathrm{pH}>7\left(\mathrm{p} K_{\mathrm{a}}=7.3 \pm 0.6\right)$ and results in a decrease in $K_{\mathrm{SV}}$.

For procaspase-3(C163S/V266H) (Figure 4B), the data are similar to those of procaspase-3(C163S) (19) and are described by two transitions. Between $\mathrm{pH} 3$ and 5, $K_{\mathrm{SV}}$ decreases from $\sim 23$ to $\sim 13$, with a $\mathrm{p} K_{\mathrm{a}}$ of $3.7 \pm 0.3$. The second transition occurs at $\mathrm{pH}>6.5$, where $\mathrm{p} K_{\mathrm{a}}=7.2 \pm 0.3$, and results in a decrease in $K_{\mathrm{SV}}$. Thus, as for the procaspase-3(C163S), the accessibility of the tryptophanyl residues to iodide increases as the $\mathrm{pH}$ is decreased. The data for the mature caspase-3(V266H) also are described by two transitions (Figure 4B). Initially, $K_{\mathrm{SV}}$ decreases between $\mathrm{pH} 3$ and $5\left(\mathrm{p} K_{\mathrm{a}}=4.1 \pm 0.1\right)$ from $\sim 23$ to $\sim 10$. The second transition occurs at $\mathrm{pH}>6\left(\mathrm{p} K_{\mathrm{a}}=6.5 \pm 0.2\right)$ and results in a further decrease in $K_{\mathrm{sv}}$.

The results of iodide quenching can be interpreted as the unraveling at lower $\mathrm{pH}$ and solvent exposure of loop L3, containing W206, which would be consistent with the position of this loop in procaspase-7 $(1,2)$ (see Figure 1A). This was examined using acrylamide as a quenching agent (Figure 4C). The results show that there is no difference between procaspase-3(C163S), procaspase-3(C163S/V266H), and caspase-3, where values of $K_{\mathrm{SV}}$ were $\sim 3-4$. Acrylamide was slightly more effective for quenching of procaspase-3(C163S/ $\mathrm{V} 266 \mathrm{E})$, the $K_{\mathrm{SV}}$ being $\sim 5$. The data are compared to those of caspase- 3 unfolded in buffer containing $8 \mathrm{M}$ urea. Under those conditions, the protein is unfolded, and the tryptophanyl residues are exposed to solvent. Importantly, the values of $K_{\mathrm{SV}}$ did not change from $\mathrm{pH} 7$ to 3 (not shown). The results shown here are similar to those described for procaspase-3 and mature caspase-3 (19), where we showed that there is no $\mathrm{pH}$-dependent change in $K_{\mathrm{SV}}$ using acrylamide or cesium as a quenching agent. This further suggests that loop L3 is not unraveled at the lower $\mathrm{pH}$. Although the precise groups have not yet been identified, the results for all proteins described here suggest that the titration at the lower $\mathrm{pH}$ affects the electrostatic environment of the tryptophanyl residues rather than unraveling loop L3. That is, the results are consistent with the protonation of an acidic group(s) at lower $\mathrm{pH}$, which lowers the negative charge(s) around the tryptophanyl residues and consequently results in an increased level of quenching by the negatively charged iodide ion. Overall, the data suggest that the $\mathrm{V} 266 \mathrm{H}$ mutation in procaspase-3 has minimal effects on the environments of the two tryptophanyl residues; however, the environments likely differ in mature caspase-3(V266H) and the wild-type protein. In contrast, the V266E mutation results in a quenching pattern in which the procaspase mutant resembles that of the mature wild-type caspase. While the catalytic efficiency of the V266 mutant does not change significantly from that of the mutant zymogen (Table 2), the quenching studies suggest that the zymogen does undergo a conformational change upon maturation. This is consistent with a decrease in the $\mathrm{p} K_{\mathrm{a}}$ of one of the catalytic residues (Figure 2B and Table 2).

\section{Tryptophan Fluorescence as a Function of pH}

Fluorescence emission scans of the V266E and V266H interface mutants were examined over the $\mathrm{pH}$ range of 2.5-9, and the average emission wavelength $(16),\langle\lambda\rangle$, was calculated at each $\mathrm{pH}$ (Figure 5). For procaspase-3(C163S/V266H) (Figure 5A), $\langle\lambda\rangle$ is constant at $343 \mathrm{~nm}$ between $\mathrm{pH} 5$ and 9 . Below $\mathrm{pH} 5$, there is a cooperative decrease in $\langle\lambda\rangle$ such that at $\mathrm{pH}<4$, $\langle\lambda\rangle$ equals $340.8 \mathrm{~nm}$. This represents a blue shift in the fluorescence emission and is further evidence that loop L3, containing W206, is not unraveled and solvent-exposed at low pH. The results for this protein are nearly identical with those of procaspase-3(C163S), described 
previously (19), except that a small inflection at $\mathrm{pH} 4(\langle\lambda\rangle=341.4 \mathrm{~nm})$ is missing in the interface mutant. The $\mathrm{p} K_{\mathrm{a}}$ that describes the single transition is $4.7 \pm 0.04$. The results for procaspase-3(C163S/V266E) in this assay were surprising. At $\mathrm{pH}>8$, the mutant reaches a maximum in $\langle\lambda\rangle$ of $344.6 \mathrm{~nm}$ (Figure 5A), the same as for the mature caspase-3 (shown in Figure 5B). Between pH 8 and 5, there is a cooperative decrease in $\langle\lambda\rangle$ to $343 \mathrm{~nm}$, which is the same $\langle\lambda\rangle$ as that of procaspase-3(C163S) or procaspase-3(C163S/V266H). The $\mathrm{p} K_{\mathrm{a}}$ for the transition is $7.6 \pm 0.2$. Between $\mathrm{pH} 5$ and $\sim 3$, there was a second cooperative decrease in $\langle\lambda\rangle$ to $341.2 \mathrm{~nm}$. The $\mathrm{p} K_{\mathrm{a}}$ for this transition is $4.1 \pm 0.1$.

The results for the wild-type mature caspase-3 are described by two transitions (Figure 5B). Between $\mathrm{pH} 7$ and $9,\langle\lambda\rangle$ reaches a maximum of $344.6 \mathrm{~nm}$. Below $\mathrm{pH} 7$, there is a cooperative decrease in $\langle\lambda\rangle$ to $341.2 \mathrm{~nm}$ at $\mathrm{pH} 4$. A second cooperative transition occurs below $\mathrm{pH} 4$ that results in an increase in $\langle\lambda\rangle$, representing a red shift in the fluorescence emission so that $\langle\lambda\rangle=342.6 \mathrm{~nm}$. The data were fit to eq 3 , and the following $\mathrm{p} K_{\mathrm{a}}$ values were determined for the two transitions: $\mathrm{p} K_{\mathrm{a} 1}=3.0 \pm 0.2$ and $\mathrm{p} K_{\mathrm{a} 2}=5.7 \pm 0.1$. The data for caspase- 3 are given in ref 19 and are shown here for comparison with those of the interface mutant. As with the procaspase-3(C163S/V266E), the results for the mature caspase-3(V266E) were surprising (Figure 5B). At $\mathrm{pH}>8$, the mutant reaches a maximum in $\langle\lambda\rangle$ of $344.6 \mathrm{~nm}$, the same as for the wild-type caspase-3. Between $\mathrm{pH} 8$ and 5, there is a cooperative decrease in $\langle\lambda\rangle$ to $343 \mathrm{~nm}$, which is the same $\langle\lambda\rangle$ as that of procaspase-3(C163S) or procaspase- $3(\mathrm{C} 163 \mathrm{~S} / \mathrm{V} 266 \mathrm{H})$. The $\mathrm{p} K_{\mathrm{a}}$ for the transition is $7.0 \pm 0.2$, which is significantly lower than that determined for the procaspase-3 interface mutant (Figure $5 \mathrm{~A}$ ). Between $\mathrm{pH} 5$ and $\sim 4$, there was a second cooperative decrease in $\langle\lambda\rangle$ to $341.2 \mathrm{~nm}$. The $\mathrm{p} K_{\mathrm{a}}$ for this transition is $4.3 \pm 0.4$. This represents a large decrease in $\mathrm{p} K_{\mathrm{a}}$ when compared to that of the mature caspase-3 (5.7 vs 4.3). In caspase-3(V266E), a third transition occurred below $\mathrm{pH} 4$ that resulted in an increase in $\langle\lambda\rangle$ to $\sim 343 \mathrm{~nm}$, similar to that observed for the wild-type caspase- 3 . The $\mathrm{p} K_{\mathrm{a}}$ for this transition is $3.0 \pm 0.2$.

As we note in ref 19, the results obtained at the lower end of the $\mathrm{pH}$ range (2.4-4) had no significant impact with respect to the main conclusions reached in this study as well as that of the accompanying paper. However, the broader $\mathrm{pH}$ range allows the calculation of the $\mathrm{p} K_{\mathrm{a}}$ values, even though at this point the responsible groups are unknown. Because of the reversibility of the transitions as well as other factors (19), we have suggested that the conformational changes described by these transitions do not result in the complete solvent exposure of the tryptophans, as would be observed in the unfolded protein. Overall in this study, the data for the V266E mutants show that depending on the $\mathrm{pH}$ range, the mutants display average emission wavelengths similar to those of the mature caspase- 3 or of the zymogen. In contrast, procaspase-3(C163S/V266H) closely resembles the procaspase-3.

\section{Circular Dichroism Spectra}

We examined the circular dichroism spectra of the interface mutants to determine whether the mutations had global effects on the protein structure aside from those described above for the active site loops, and the data are compared to those of procaspase-3(C163S) and mature caspase-3 (Figure 6). The data show that upon maturation, the magnitude of the farUV CD signal decreases (Figure 6A). At this point, it is not clear whether these changes result from a decrease in the level of secondary structure upon maturation or from changes in the tertiary structure, which may also affect the signal in this region. Both processes are known to occur upon maturation $(1,2)$. The results also are consistent with the loss of the propeptide upon maturation, as was observed previously with the "pro-less" variant of procaspase-3 (7). The data show that while the magnitude of the far-UV CD signal decreases upon maturation, the magnitude of the near-UV CD signal increases, again consistent with conformational changes upon maturation. 
The far-UV CD signals of the procaspase interface mutants (Figures 6B,C) were somewhat lower than that of procaspase-3(C163S) (Figure 6A). In addition, only the magnitude of the signal of the V266E mutant decreased upon maturation (Figure 6B). In the near-UV region, the procaspase mutants in general have a larger signal magnitude than procaspase-3(C163S). Upon maturation, the magnitude of the signal of the V266E mutant (Figure 6B) increased and is significantly larger than that of the wild-type caspase-3 (Figure 6A). Interestingly, the magnitude of the near-UV CD signal of the $\mathrm{V} 266 \mathrm{H}$ mutant decreased upon maturation (Figure 6C). Overall, the data show that the interface mutants are folded with well-packed tertiary structures. While it should be noted that the mutations might affect other regions of the proteins, the changes in the near-UV CD spectra are consistent with changes in the active site loops described above.

\section{DISCUSSION}

We have shown that replacing valine 266 with glutamate in the dimer interface of procaspase- 3 results in a 60 -fold increase in the activity of the procaspase. The increase in activity may be due to a change in the environment of the catalytic C163, resulting in a decrease in the $\mathrm{p} K_{\mathrm{a}}$ of this group. This effect is likely the result of stabilizing contacts in the loop bundle, including loop L4, which are mediated by movements in the intersubunit linker. The data show that the mutation affects loops L1, L3, and L4. On the basis of proteolysis and fluorescence emission studies, the V266E mutants have features of both the procaspase and of the mature caspase. For example, limited proteolysis by V8 protease suggests a conformational change that affects loop L4 (E248/D253), the intersubunit linker (E173/E190), and the region of E98/E106. In the mature caspase-3, residue E106 interacts with R86 to form a salt bridge. Residue R86 is on the outside-most strand, $\beta$-strand 2 , the edge of which is exposed to solvent. These contacts do not occur in procaspase-3, and consequently, the procaspase is cleaved by V8 protease at E106 (19). Overall, the data for procaspase-3(C163S/V266E) show that loop L4 is protected from cleavage by V8 protease, that the contacts between E106 and R86 may have formed in the procaspase, and that loop L2' (E173/D190) is less accessible for cleavage by V8 protease. The latter two points are observed in mature caspase-3. The lack of cleavage at D190 in the V266E mutant is interesting because the exposed loop L2' (intersubunit linker) in procaspase-3 becomes less accessible to V8 protease after zymogen processing. In this case, the loop flips $180^{\circ}$ so that it makes new contacts with loops L2 and L4 of the second monomer. Because loop L2' is covalently connected to loop L2 in the procaspase, preventing its flipping, it is not clear why E173/D190 would be protected in procaspase-3(C163S/V266E). In addition to these effects, loop L1 (K57/R64) is less accessible to cleavage by trypsin and loop L3 (R207) is protected from cleavage.

A comparison of the data in Figure 5 shows that, at $\mathrm{pH}>7$, the fluorescence emission is redshifted upon maturation of the caspase. We suggest that the red shift results from movements of loop L4 away from the active site and subsequent formation of the loop bundle. At physiological $\mathrm{pH}$, this represents the major conformational change in the protein upon maturation, and the loop bundle cannot form in the procaspase. The movements are consistent with a more solvent-accessible active site as shown by the proteolysis studies and the enzyme activity measurements. If this transition represents formation of the loop bundle, then the data suggest that contacts in the loop bundle must form, at least partially, in the V266E mutants. The contacts appear to be stabilized by ionic interactions and are weaker than in caspase-3, so at $\mathrm{pH} 6.5$ the bundle resembles that of the procaspase-3. The weaker contacts in the loop bundle may also explain the lower activity of caspase-3(V266E) compared to that of wild-type caspase-3 (Table 2). 
In contrast to V266E, replacing V266 with histidine abolishes activity in the procaspase and in the mature caspase. As shown in Figure 3, the V266H mutation may result in a hyperexposed R207, which is cleaved rapidly by trypsin. On the basis of the proteolysis and fluorescence emission studies (Figures 3-5), the V266H mutation does not appear to affect loops L1 and L4 or the intersubunit linker. Loop L3 forms the bulk of the substrate binding pocket; thus, the hyperexposed R207, on loop L3, is consistent with the lack of enzyme activity.

While the mechanism remains unknown, the effect of V266H occurs over long distances ( $20 \AA$ between V266 and R207). We suggest that the effects of H266 may be mediated through Y197. Upon maturation, the side chain of Y197 rotates away from the active site and toward the dimer interface (see Figure 7). Modeling studies with H266 in the interface suggest steric clashes exist between the side chains of H266 and Y197, which may prevent the proper rotation of Y197. This mechanism is observed in caspase-9, which contains a phenylalanine at position 404 (comparable to residue 266 in caspase-3). In that case, the presence of two phenylalanyl side chains in the interface prevents proper positioning of Y197, and this results in only one intact active site rather than two. Aside from an increase in side chain volume between valine and histidine, the effect of a positive charge on H266 cannot be ruled out at this point.

Although the mechanism of the loop movements is not clear, we suggest that the effects of V266E are mediated through contacts with R164, on loop L2. During maturation of the caspase, R164 rotates away from the active site and points toward the dimer interface (Figure 7). The side chain of R164 intercalates between Y197 and P201, on the "elbow loop", and these contacts appear to stabilize loop L3. The positive charge of R164 is neutralized by E124, which resides on a loop above the interface. This loop is important because it contains H121, part of the catalytic dyad, and G122, which forms part of the oxyanion hole. We suggest that the V266E mutation positions the E266 side chain so that it interacts with the guanidinium group of R164. Indeed, these contacts are observed in caspase-1, where E390, the comparable position in the interface, is within $2.5 \AA$ of the arginine side chain (18). In caspase-3, it is not clear how the putative interactions between R164 and E266 might affect movements in the intersubunit linker and in loop L4. The mechanisms of these movements await further study. Nevertheless, it is remarkable to conclude that a point mutation in the dimer interface of procaspase- 3 affects the conformations of three of the five active site loops and results in a significant increase in enzyme activity.

\section{Acknowledgments}

We thank Erin Shiver for cloning of procaspase-3(C163S/V266E). We also thank Dr. Stuart Maxwell and Elizabeth Tran for their assistance with immunoassays and Kakoli Bose for assistance with mass spectrometry and for helpful discussions.

\section{REFERENCES}

1. Chai J, Wu Q, Shiozaki E, Srinivasula SM, Alnemri ES, Shi Y. Cell. 2001; 107:399-407. [PubMed: 11701129]

2. Riedl SJ, Fuentes-Prior P, Renatus M, Kairies N, Krapp S, Huber R, Salvesen GS, Bode W. Proc. Natl. Acad. Sci. U.S.A. 2001; 98:14790-14795. [PubMed: 11752425]

3. Shi Y. Mol. Cell. 2002; 9:459-470. [PubMed: 11931755]

4. Roy S, Bayly CI, Gareau Y, Houtzager VM, Kargman S, Keen SLC, Rowland K, Seiden IM, Thornberry NA, Nicholson DW. Proc. Natl. Acad. Sci. U.S.A. 2001; 98:6132-6137. [PubMed: 11353841]

5. Bose K, Clark AC. Biochemistry. 2001; 40:14236-14242. [PubMed: 11714277] 
6. Thornberry NA, Bull HG, Calaycay JR, Chapman KT, Howard AD, Kostura MJ, Miller DK, Molineaux SM, Weidner JR, Aunins J, Elliston KO, Ayala JM, Casano FJ, Chin J, Ding GJ-F, Egger LA, Gaffney EP, Limjuco G, Palyha OC, Raju SM, Rolando AM, Salley JP, Yamin T-T, Lee TD, Shively JE, MacCross M, Mumford RA, Schmidt JA, Tocci MJ. Nature. 1992; 356:768-774. [PubMed: 1574116]

7. Pop C, Chen Y-R, Smith B, Bose K, Bobay B, Tripathy A, Franzen S, Clark AC. Biochemistry. 2001; 40:14224-14235. [PubMed: 11714276]

8. Hedegs, J.; Sarrafzadeh, S.; Lear, JD.; McRorie, DK. Modern Analytical Ultracentrifugation. Shuster, T.; Laue, TM., editors. Birkhauser; Boston: 1984.

9. Johnson ML, Correira JJ, Yphantis DA, Halvorson HR. Biophys. J. 1981; 36:575-588. [PubMed: 7326325]

10. Davies GE, Stark GR. Proc. Natl. Acad. Sci. U.S.A. 1970; 66:651-656. [PubMed: 4913206]

11. Tobin H, Staehelin T, Gordon J. Proc. Natl. Acad. Sci. U.S.A. 1979; 76:4350-4354. [PubMed: 388439]

12. Stennicke HR, Salvesen GS. Methods. 1999; 17:313-319. [PubMed: 10196102]

13. Stennicke HR, Salvesen GS. J. Biol. Chem. 1997; 272:25719-25723. [PubMed: 9325297]

14. Tang S-S, Chang G-G. Biochem. J. 1996; 315:599-606. [PubMed: 8615835]

15. Lakowicz, JR. Principles of fluorescence spectroscopy. Plenum Press; New York: 1983.

16. Royer CA, Mann CJ, Matthews CR. Protein Sci. 1993; 2:1844-1852. [PubMed: 8268795]

17. Lewis SD, Johnson FA, Shafer JA. Biochemistry. 1981; 20:48-51. [PubMed: 7470479]

18. Walker NPC, Talanian RV, Brady KD, Dang LC, Bump NJ, Ferenz CR, Franklin S, Ghayur T, Hackett MC, Hammill LD, Herzog L, Hugunin M, Houy W, Mankovich JA, McGuiness L, Orlewicz E, Paskind M, Pratt CA, Reis P, Summani A, Terranova M, Welch JP, Xiong L, Moller A, Tracey DE, Kamen R, Wong WW. Cell. 1994; 78:343-352. [PubMed: 8044845]

19. Bose K, Pop C, Feeney B, Clark AC. Biochemistry. 2003; 42:12298-12310. [PubMed: 14567691] 


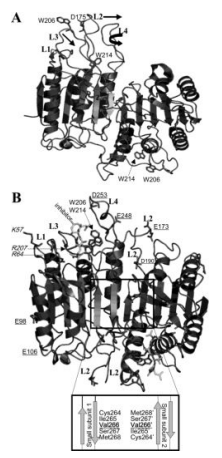

Figure 1.

Structure of procaspase-7 (PDB entry 1GQF) (A) with positions of four active site loops (L1-L4). Residues W206 (caspase-3 numbering), in loop L3, W214, at the base of the active site, and D175, the site of processing, are shown. The black arrows show the direction of the loop movements upon maturation. (B) Structure of caspase-3 (PDB entry 1CP3) with positions of the catalytic loops after processing. The residues cleaved by trypsin (italic) and V8 protease (underlined) are shown. The caspase- 3 heterodimer interface corresponds to the boxed region, and amino acids in the interface are shown below the structure. The prime indicates residues from the second monomer (A) or heterodimer (B). Structures were generated using PyMol (Delano Scientific). 

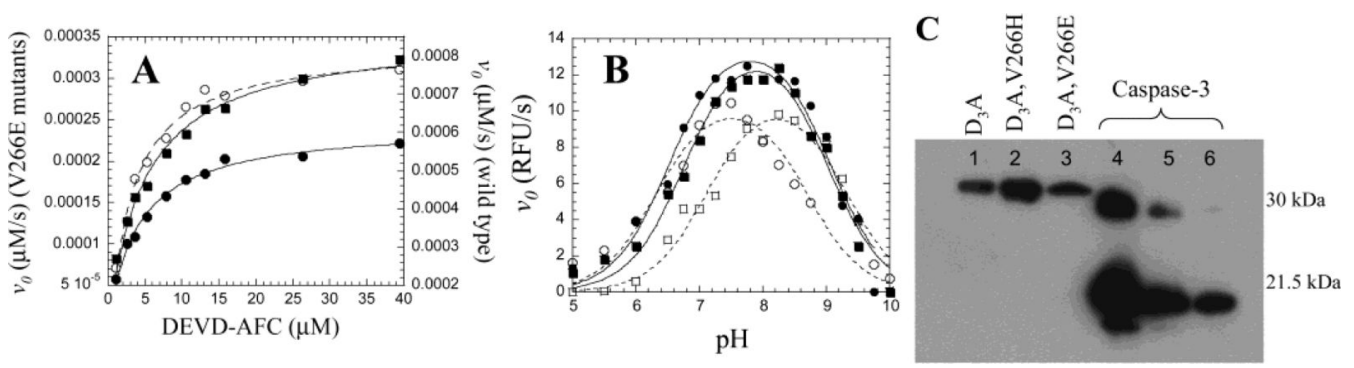

Figure 2.

(A) Plot of initial velocity $\left(v_{0}\right)$ vs substrate concentration for caspase-3(V266E) (•), procaspase-3( $\left.\mathrm{D}_{3} \mathrm{~A} / \mathrm{V} 266 \mathrm{E}\right)(\mathbf{\square})$, and caspase-3 (o). The lines represent fits of the data to the Michaelis-Menten equation. (B) Effect of $\mathrm{pH}$ on initial velocity $\left(v_{0}\right)$ for caspase-3(V266E) $(\bullet)$, procaspase-3( $\left.\mathrm{D}_{3} \mathrm{~A} / \mathrm{V} 266 \mathrm{E}\right)(\boldsymbol{\bullet})$, caspase-3 (o), and procaspase-3( $\left.\mathrm{D}_{3} \mathrm{~A}\right)(\square)$. The solid and dashed lines represent fits of the data to eq 1 . For clarity, all data were scaled to match the concentration of procaspase- $3\left(\mathrm{D}_{3} \mathrm{~A}\right)$ so that the plot corresponds to the following final protein concentrations: $\sim 0.75 \mathrm{nM}$ caspase-3, $\sim 1.6 \mathrm{nM}$ V266E mutants, and $10 \mathrm{nM}$ procaspase- $3\left(\mathrm{D}_{3} \mathrm{~A}\right)$. The parameters obtained from the fits of the data in panels $\mathrm{A}$ and $\mathrm{B}$ are presented in Table 2. (C) Anti-caspase-3 immunoblot of procaspase-3( $\mathrm{D}_{3} \mathrm{~A}$ ) (lane 1, 500

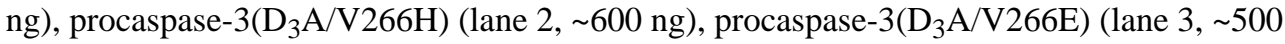
ng), and caspase-3 (lanes 4-6, 670, 67, and $13 \mathrm{ng}$, respectively). 


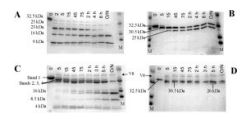

Figure 3.

Trypsin digests of procaspase-3(C163S/V266H) (A) and procaspase-3(C163S/V266E) (B). Times of incubation (in minutes or hours) are shown above each panel. The $32 \mathrm{kDa}$ band represents the full-length protein, whereas the 25,23,16, and $9 \mathrm{kDa}$ bands are the cleavage products as described in the text. $(\mathrm{C}$ and $\mathrm{D}) \mathrm{V} 8$ protease digests of procaspase- $3(\mathrm{C} 163 \mathrm{~S} /$ V266H) (panel C) and procaspase-3(C163S/V266E) (panel D). Band 1 refers to the fulllength protein. Bands 2-4 refer to the 16, 8.5, and $4 \mathrm{kDa}$ fragments (C), and the 30.5 and 26 $\mathrm{kDa}$ fragments (D) are the cleavage products as described in the text. M refers to molecular mass markers. The position of V8 protease is indicated by the arrows. 

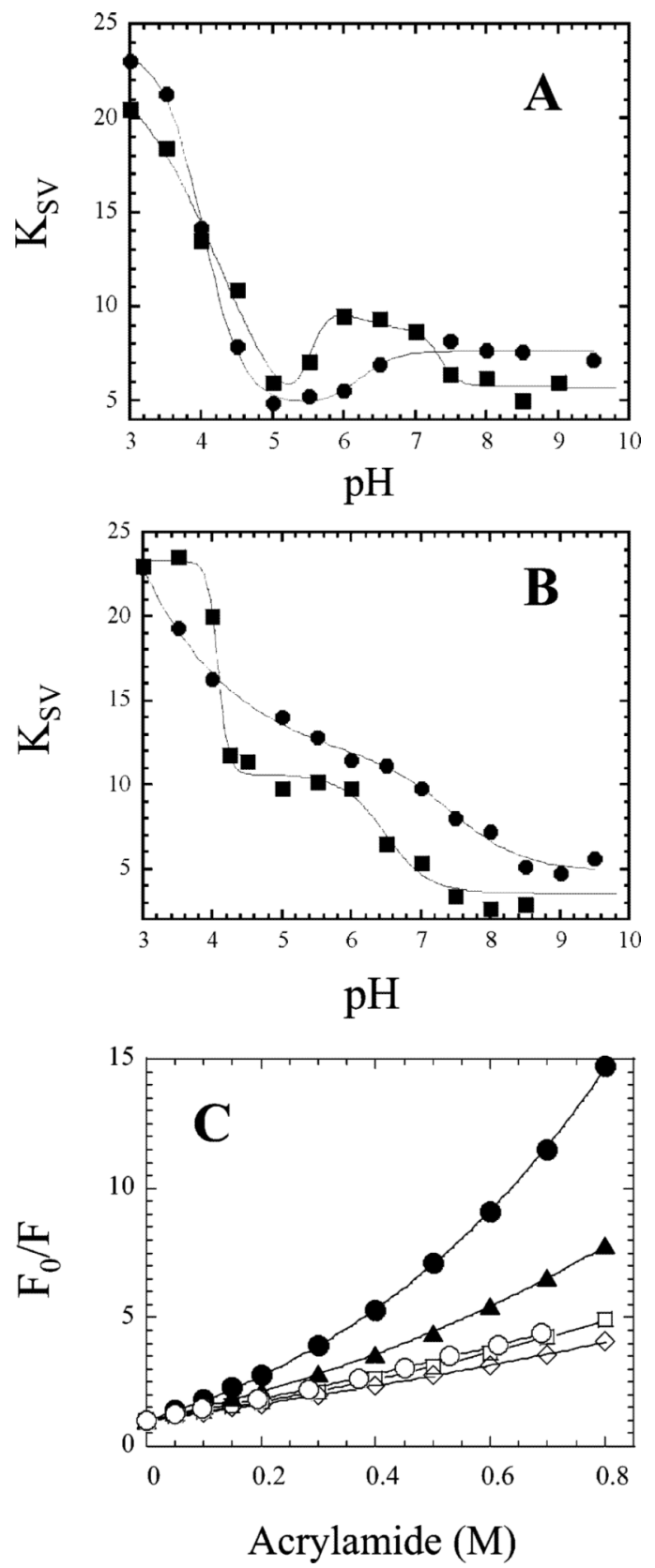

Figure 4.

(A) Stern-Volmer constants $\left(K_{\mathrm{SV}}\right)$ for $\mathrm{KI}$ quenching vs $\mathrm{pH}$ for procaspase-3(C163S/V266E) $(\bullet)$ and caspase-3(V266E) (ם). (B) Stern-Volmer constants $\left(K_{\mathrm{SV}}\right)$ for KI quenching vs pH for procaspase-3(C163S/V266H) (•) and caspase-3(V266H) (ロ). (C) Relative fluorescence $\left(F_{0} / F\right)$ vs acrylamide concentration at $\mathrm{pH} 7.2$. For panel $\mathrm{C}$, the symbols represent the following: caspase-3 ( $\square)$, procaspase-3(C163S) (o), procaspase-3(C163S/V266H) $(\diamond)$, procaspase-3(C163S/V266E) $(\boldsymbol{\Delta})$, and caspase-3 in $8 \mathrm{M}$ urea $(\bullet)$. Solid lines represent fits of the data to eq 2 (panels A and B) or the Stern-Volmer equation (panel C) as described in Materials and Methods. Parameters obtained from the fits are described in the text. 

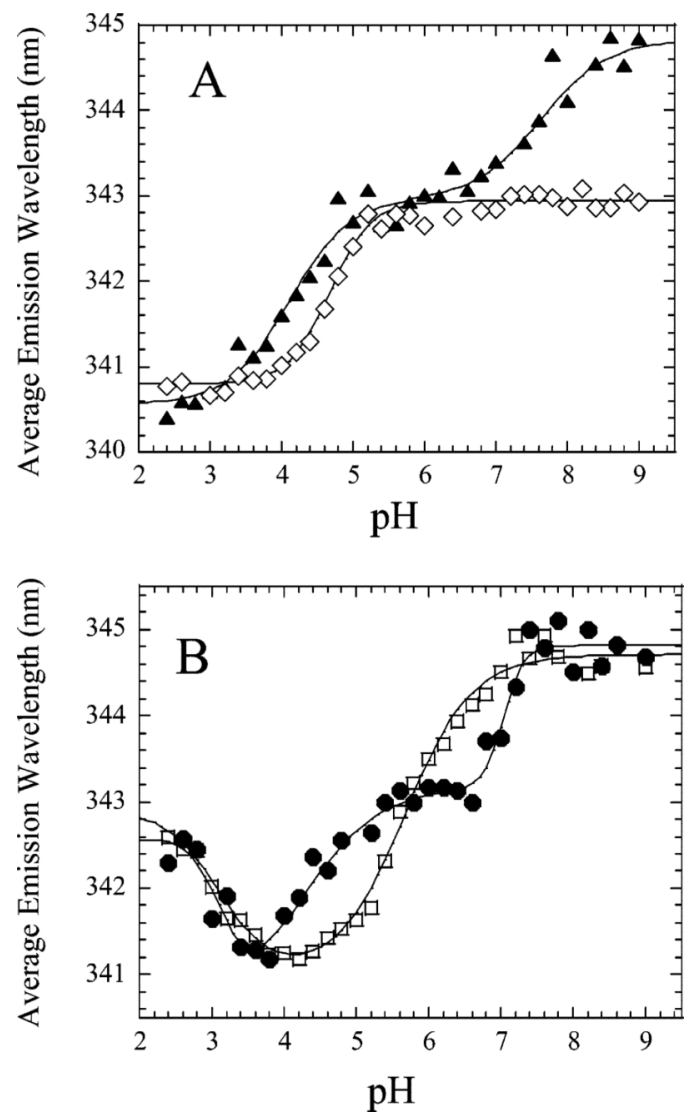

Figure 5.

Average emission wavelength $(\langle\lambda\rangle)$ vs $\mathrm{pH}$ for procaspase-3(C163S/V266H) $(\diamond)$ and

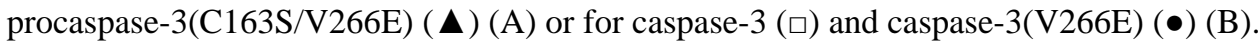
Solid lines represent fits of the data to eq 3 as described in Materials and Methods. Parameters obtained from the fits are described in the text. 

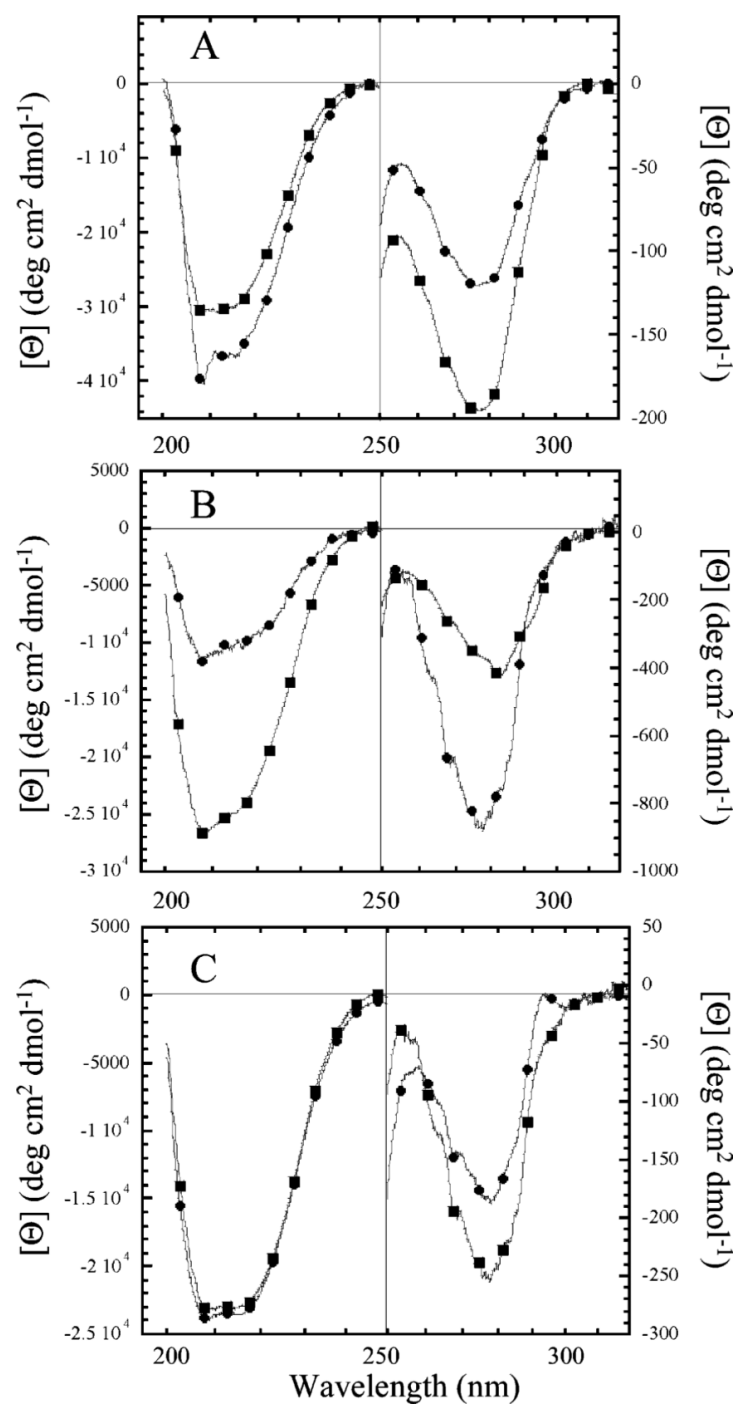

Figure 6.

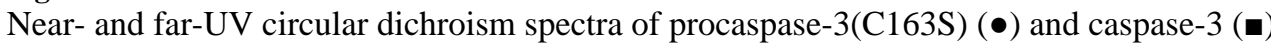

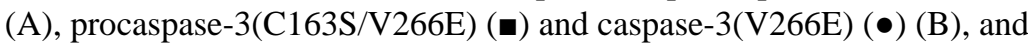
procaspase-3(C163S/V266H) $(\bullet)$ and caspase-3(V266H) $(\bullet)(\mathrm{C})$. The experiments were performed in a buffer containing $50 \mathrm{mM} \mathrm{KH} 2-\mathrm{PO}_{4} / \mathrm{K}_{2} \mathrm{HPO}_{4}(\mathrm{pH} 7.5)$ and $1 \mathrm{mM}$ DTT, and protein concentrations were between 15 and $40 \mu \mathrm{M}$. 


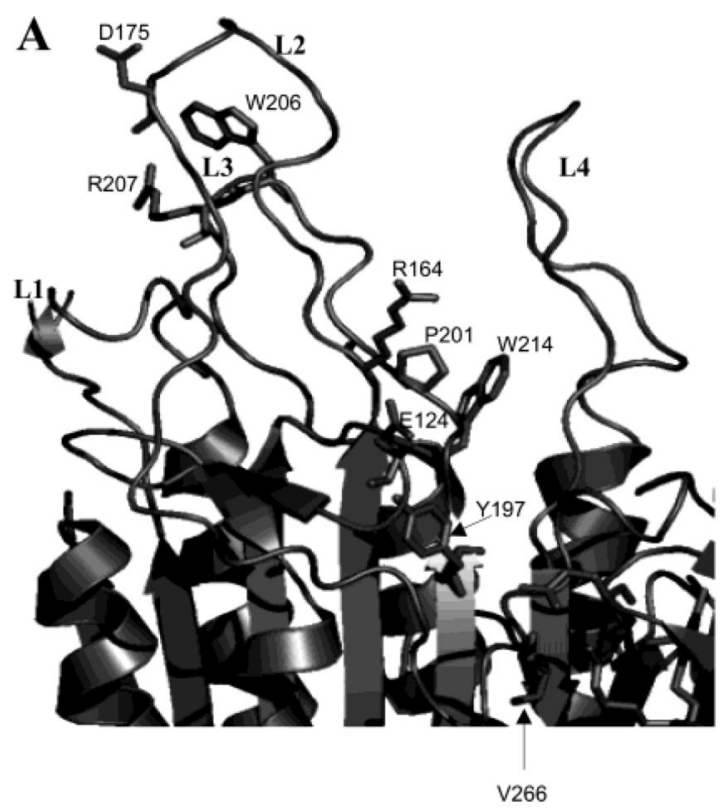

B

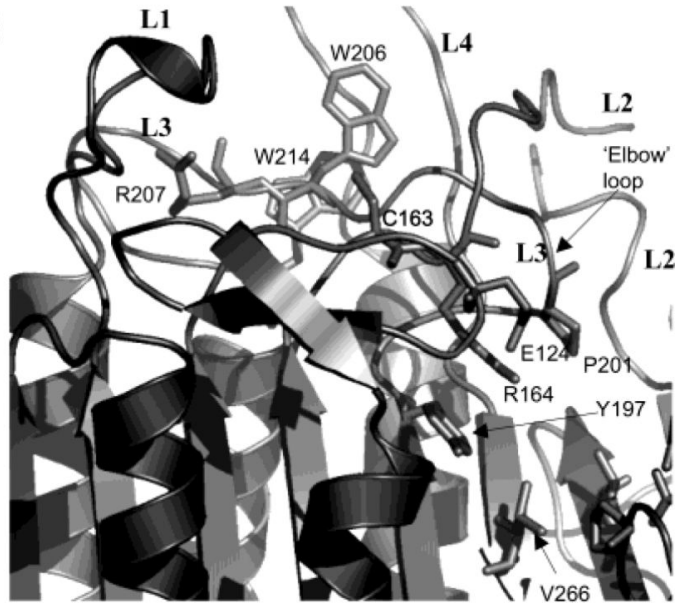

Figure 7.

(A) Structure of procaspase-7 highlighting the location of R164, E124, Y197, P201, and V266 before processing. (B) Caspase-3 structure highlighting the positions of the elbow loop (P201), R164, E124, Y197, and V266 after processing. The caspase inhibitor has been removed from the figure for clarity. Structures were generated using PyMol (Delano Scientific). 
Table 1

Calculated and Experimental Molecular Masses for (Pro)Caspase-3 Interface Mutants

\begin{tabular}{lcc}
\hline & calculated molecular mass (Da) & experimental molecular mass (Da) \\
\hline procaspase-3(C163S/V266E) & 32568 & 65667 \\
procaspase-3(C163S/V266H) & 32676 & 67350 \\
caspase-3(V266E) & 29688 & 63308 \\
caspase-3(V266E) and Z-VAD-FMK & 30123 & 63690 \\
\hline
\end{tabular}


